\title{
Failure of the Factor Theorem for Borel pre-Hilbert spaces
}

\author{
by \\ Tadeusz Dobrowolski (Pittsburg, KS) and \\ Witold Marciszewski (Warszawa)
}

\begin{abstract}
In every infinite-dimensional Fréchet space $X$, we construct a linear subspace $E$ such that $E$ is an $F_{\sigma \delta \sigma}$-subset of $X$ and contains a retract $R$ so that $R \times E^{\omega}$ is not homeomorphic to $E^{\omega}$. This shows that Toruńczyk's Factor Theorem fails in the Borel case.
\end{abstract}

1. Introduction. In [Tor1] and [Tor2] Toruńczyk has proved that for retracts $R$ of certain metric linear spaces $E$ the product $R \times E$ is homeomorphic to $E$, the fact referred to as the Factor Theorem. In particular, he showed that the Factor Theorem holds for all infinite-dimensional spaces $E$ that are locally convex and completely metrizable, and for the class of incomplete spaces $E$ that admit a certain weak product structure. The Factor Theorem for more general classes of incomplete metric linear spaces $E$ can be deduced from the results on absorbing sets (e.g., [BM] and [BRZ]; see also Section 6). The Factor Theorem led to the following natural question of Toruńczyk (see [Tor1, p. 60], [Ge] and [DM1]).

1.1. Problem. For a retract $R$ of an infinite-dimensional locally convex metric linear space $E$, is $R \times E^{\omega}$ homeomorphic to $E^{\omega}$ ?

It was shown in [Tor2] that the answer is affirmative if $R$ is completely metrizable. On the other hand, there are separable Baire pre-Hilbert spaces $E$ with first category closed linear subspaces $R$. Then $R$ is a retract of $E$ but $R \times E^{\omega}$ is of the first category, while $E^{\omega}$ is of the second category, so Problem 1.1 has a negative solution. Consequently, the assertion of the Factor Theorem is violated because if $R \times E$ were homeomorphic to $E$ then $R \times E^{\omega}$ would be homeomorphic to $E^{\omega}$. We learned about that phenomenon from R. Pol several years ago, and we recall his reasoning in Section 6 .

2000 Mathematics Subject Classification: 57N17, 46C05.

Key words and phrases: $Z_{\sigma}$-space, Fréchet space, pre-Hilbert space, Borel set, retract.

The second author was supported in part by KBN grant 2 P03A 01115. 
Another implicit example of that sort can also be found in $[\mathrm{MvM}]$. However, in that approach $E$ had to be non-Borel, for otherwise it would be complete by the classical theorem of Banach. This left unanswered the question of whether the Factor Theorem holds true, or whether Problem 1.1 has an affirmative solution, for Borel metric linear spaces. These questions were repeated on several occasions [DM1, Problems: 603, 605, and 606], and in [We, LS14].

The aim of this paper is to show that Problem 1.1 has a negative answer for a certain linear subspace $E \subset \ell_{2}$ that is an $F_{\sigma \delta \sigma}$-set. This solves the above-mentioned problems of [DM1, We] and demonstrates that the Factor Theorem fails for Borel spaces. Our space $E$ has the property that $E^{\omega}$ is not a $Z_{\sigma}$-space while $E$ contains a closed topological copy $R$ of an absolute retract $\sigma$, the subspace of $[0,1]^{\omega}$ consisting of all eventually zero sequences. The set $R$ is a retract of $E$. Since $R$ is a $Z_{\sigma}$-space, so is $R \times E^{\omega}$, hence it cannot be homeomorphic to $E^{\omega}$. Actually, it suffices to take for $E$ the space $L=\operatorname{span}(\mathcal{E})$, where $\mathcal{E}=\left\{\left(x_{n}\right) \in \ell_{2}:(\forall n)\left[x_{n} \in \mathbb{Q}\right]\right\}$ is the well known Erdös space. This space $L$ was the first example of a Borel infinitedimensional linear space which is not a $Z_{\sigma}$-space, given by T. Banakh (see [BRZ] and [Ba]). Let us mention that spaces of the form $\operatorname{span}(\mathfrak{E})$, for some Erdős-like subsets $\mathfrak{E}$, were used in the theory of locally convex Baire-like spaces to construct barrelled normed spaces of the first category (see $[\mathrm{Ku}$, $\S 5.3],[\mathrm{PCB}]$ or $[\mathrm{Va}])$.

For some technical reasons, we have chosen to work with a slightly different construction, which involves a certain Borel linear subspace $E_{\Phi}$ of a Fréchet space $X$ that is associated with an arbitrary biorthogonal sequence in $X$; see Section 2. The Borel complexity of the spaces $E_{\Phi}$ is discussed in Section 3. Here we also give an exact evaluation of the Borel class of the Banakh example $L$. In Section 4, we investigate the factorization properties of our spaces $E_{\Phi}$. Section 5 contains some related comments on $Z_{\sigma}$-property in products. In Section 6, we provide other results and examples related to the Factor Theorem; among other things, we give an affirmative answer to Problem 1.1 for $\sigma$-compact spaces $E$.

Acknowledgments. The authors would like to thank the referee for his insightful report and valuable suggestions. In particular, his suggestions were incorporated in $4.3,6.2,6.3$, and 6.6.

2. Linear spaces $E_{\Phi}$ associated with biorthogonal systems $\Phi$. Recall that by a Fréchet space we mean a completely metrizable locally convex topological linear space. For the notions from infinite-dimensional topology that we are using, we refer the reader to $[\mathrm{vM}]$. By $\mathbb{N}$ and $\mathbb{Q}$ we denote the set of positive integers and the set of rationals, respectively. 
Quite often we will treat the real line $\mathbb{R}$ as a linear space over the field $\mathbb{Q}$. Having this in mind, for a set $A \subset \mathbb{R}$, by $\operatorname{dim}_{\mathbb{Q}}(A)$ we denote the linear dimension of the subspace $\operatorname{span}(A)$ of the linear space $\mathbb{R}$.

Let $X$ be a Fréchet space, $\left(x_{n}\right)_{n \in \mathbb{N}}$ a sequence of vectors in $X$, and $\left(\varphi_{n}\right)_{n \in \mathbb{N}}$ a sequence of continuous linear functionals on $X$. Let us recall that the system $\Phi=\left(\left(\varphi_{n}\right),\left(x_{n}\right)\right)$ is biorthogonal if $\varphi_{n}\left(x_{k}\right)=0$ for all $n \neq k$, and $\varphi_{n}\left(x_{n}\right)=1$ for every $n$. A slight modification of the standard proof of the existence of biorthogonal systems in infinite-dimensional Banach spaces (see [LT, p. 43]) shows the following:

2.1. Proposition. In every infinite-dimensional Fréchet space there exists a biorthogonal system.

Given a biorthogonal system $\Phi$ in a Fréchet space $X$, we will consider the following subsets of $X$ :

$$
\begin{aligned}
Q_{\Phi} & =\left\{x \in X: \varphi_{n}(x) \in \mathbb{Q} \text { for all } n \in \mathbb{N}\right\}, \\
E_{\Phi} & =\left\{x \in X: \operatorname{dim}_{\mathbb{Q}}\left(\left\{\varphi_{n}(x): n \in \mathbb{N}\right\}\right)<\infty\right\} .
\end{aligned}
$$

Obviously $E_{\Phi}$ is a linear subspace of $X$ containing $Q_{\Phi}$.

If $X$ is a sequence Fréchet space (e.g., $X=\ell_{2}$ or $\mathbb{R}^{\omega}$ ) then the standard example of a biorthogonal system in $X$ is the system $\Phi=\left(\left(p_{n}\right),\left(e_{n}\right)\right)$, where $p_{n}$ is the projection onto the $n$th axis and $e_{n}$ is the $n$th unit vector in $X$.

Let $\mathcal{E}$ be the Erdős space defined in the Introduction and let $L=\operatorname{span}(\mathcal{E})$. We have $\mathcal{E}=Q_{\Phi}$ and $L \subset E_{\Phi}$, where $\Phi$ is the standard biorthogonal system in $\ell_{2}$ described above. Below we show that the Banakh example $L$ is different from our space $E_{\Phi}$. Observe that, for the standard biorthogonal system $\Phi$ in the countable product $\mathbb{R}^{\omega}$ of real lines, we have $E_{\Phi}=\operatorname{span}\left(Q_{\Phi}\right)$.

2.2. Proposition. The Banakh example $L$ is a proper subspace of $E_{\Phi}$.

Proof. We use the standard fact that there exist sequences $\left(k_{n}\right)$ and $\left(l_{n}\right)$ of natural numbers such that $a_{n}=k_{n} \sqrt{2}-l_{n} \in(0,1 / n)$. Obviously the sequence $\left(a_{n}\right)$ belongs to the space $E_{\Phi}$. We will show that $\left(a_{n}\right) \notin L$.

Suppose on the contrary that there exist real numbers $t_{1}, \ldots, t_{m}$ and sequences $\left(q_{n}^{1}\right), \ldots,\left(q_{n}^{m}\right) \in \ell_{2}$ of rational numbers such that, for all $n \in \mathbb{N}$,

$$
a_{n}=t_{1} q_{n}^{1}+\ldots+t_{m} q_{n}^{m} .
$$

We may assume that the number $m$ above is minimal. Then the sequence $t_{1}, \ldots, t_{m}$ is linearly independent over $\mathbb{Q}$. Indeed, if $t_{i}=\sum_{j \neq i} p_{j} t_{j}$ for some $p_{j} \in \mathbb{Q}$, then we would have, for every $n \in \mathbb{N}, a_{n}=\sum_{j \neq i} t_{j}\left(q_{n}^{j}+p_{j} q_{n}^{i}\right)$, a contradiction with our assumption on $m$.

Take $n_{1}$ and $n_{2}$ such that $a_{n_{1}}>a_{n_{2}}$ and $k_{n_{1}}<k_{n_{2}}$. Let us check that $a_{n_{1}}$ and $a_{n_{2}}$ are linearly independent over $\mathbb{Q}$. If $a_{n_{1}}=q a_{n_{2}}$ for some $q \in \mathbb{Q}$, then we would have $k_{n_{1}} \sqrt{2}-l_{n_{1}}=q k_{n_{2}} \sqrt{2}-q l_{n_{2}}$. Since $q>1$ we have 
$k_{n_{1}}<q k_{n_{2}}$. Therefore, the above equation contradicts the fact that $\sqrt{2}$ and 1 are linearly independent over $\mathbb{Q}$.

It follows that $\sqrt{2}$ and 1 are linear combinations with rational coefficients of $a_{n_{1}}$ and $a_{n_{2}}$. Consequently, we can find rational numbers $p_{1}, \ldots, p_{m}$, $r_{1}, \ldots, r_{m}$ such that $\sqrt{2}=p_{1} t_{1}+\ldots+p_{m} t_{m}$ and $1=r_{1} t_{1}+\ldots+r_{m} t_{m}$. Let $M$ be a common denominator of all numbers $p_{1}, \ldots, p_{m}, r_{1}, \ldots, r_{m}$. Since $a_{n}=k_{n} \sqrt{2}-l_{n}$ we obtain

$$
a_{n}=\frac{i_{n}^{1}}{M} t_{1}+\ldots+\frac{i_{n}^{m}}{M} t_{m}
$$

for some integers $i_{n}^{j}$. From the fact that $t_{1}, \ldots, t_{m}$ are linearly independent over $\mathbb{Q}$ we infer that $q_{n}^{j}=i_{n}^{j} / M$ for every $n \in \mathbb{N}$ and $j \leq m$. For every $n$, there is a $j_{n}=j \leq m$ such that $q_{n}^{j} \neq 0$ because $a_{n} \neq 0$. It follows that, for some $j \leq m$ and infinitely many $n \in \mathbb{N}$, we have $\left|q_{n}^{j}\right| \geq 1 / M$. Hence $\left(q_{n}^{j}\right)_{n} \notin \ell_{2}$, which is a contradiction.

We do not know if the above spaces $L$ and $E_{\Phi}$ are homeomorphic. One can ask similar questions about homeomorphisms between $L$, or $E_{\Phi}$, and $E_{\Psi}$, where $\Psi$ is the standard biorthogonal system in $\mathbb{R}^{\omega}$ (cf. [Ba, Question 3]).

Let us finish this section with the following simple fact that we will frequently use in what follows (cf. [BP, p. 268]).

2.3. Lemma. Let $\Phi=\left(\left(\varphi_{n}\right),\left(x_{n}\right)\right)$ be a biorthogonal system in a Fréchet space $X$. There exists a sequence $\left(s_{n}\right)$ of positive reals such that, for every sequence $\left(t_{n}\right)$ with $t_{n} \in\left[-s_{n}, s_{n}\right]$, the series $\sum_{n \in \mathbb{N}} t_{n} x_{n}$ is convergent and we have $\varphi_{k}\left(\sum_{n \in \mathbb{N}} t_{n} x_{n}\right)=t_{k}$ for all $k \in \mathbb{N}$. Moreover, the map $\left(t_{n}\right) \mapsto \sum_{n \in \mathbb{N}} t_{n} x_{n}$ is a homeomorphic (affine) embedding of $\prod_{n \in \mathbb{N}}\left[-s_{n}, s_{n}\right]$ into $X$.

Proof. Let $d$ be a translation-invariant metric on $X$. For every $n \in \mathbb{N}$, take $s_{n} \in(0, \infty)$ such that $d\left(0, t_{n} x_{n}\right) \leq 2^{-n}$ for every $t_{n} \in\left[-s_{n}, s_{n}\right]$. Then the series $\sum_{n \in \mathbb{N}} t_{n} x_{n}$ converges. The biorthogonality of the system $\Phi$ and the continuity of $\varphi_{k}$ guarantee that $\varphi_{k}\left(\sum_{n \in \mathbb{N}} t_{n} x_{n}\right)=t_{k}$. One can easily verify that the map $\left(t_{n}\right) \mapsto \sum_{n \in \mathbb{N}} t_{n} x_{n}$ is continuous and the above equality shows that this map is injective.

\section{Borel complexity of the spaces $E_{\Phi}$}

3.1. Proposition. Let $\Phi=\left(\left(\varphi_{n}\right),\left(x_{n}\right)\right)$ be a biorthogonal system in a Fréchet space $X$. Then $E_{\Phi}$ is an $F_{\sigma \delta \sigma}$-subset of $X$.

Proof. It is clear that a vector $x \in X$ belongs to $E_{\Phi}$ if and only if there is an $n \in \mathbb{N}$ such that, for every $k>n$, the value $\varphi_{k}(x)$ is a linear combination 
over $\mathbb{Q}$ of the values $\varphi_{1}(x), \ldots, \varphi_{n}(x)$. Therefore we have

$$
\begin{aligned}
E_{\Phi}= & \{x \in X: \\
& \left.(\exists n \in \mathbb{N})(\forall k>n)\left(\exists q_{1}, \ldots, q_{n} \in \mathbb{Q}\right)\left[\varphi_{k}(x)=\sum_{i=1}^{n} q_{i} \varphi_{i}(x)\right]\right\} \\
= & \bigcup_{n \in \mathbb{N}} \bigcap_{k>n} \bigcup_{q_{1}, \ldots, q_{n} \in \mathbb{Q}}\left\{x \in X: \varphi_{k}(x)=\sum_{i=1}^{n} q_{i} \varphi_{i}(x)\right\},
\end{aligned}
$$

which shows that $E_{\Phi}$ is an $F_{\sigma \delta \sigma}$-set.

3.2. Lemma. For every sequence $\left(s_{n}\right)_{n=1}^{\infty}$ of positive reals, there exists a sequence $\left(C_{n}\right)_{n=1}^{\infty}$ of subsets of $\mathbb{R}$ satisfying

(i) $C_{n} \subset\left[0, s_{n}\right]$,

(ii) $C_{n}$ is a topological copy of the Cantor set,

(iii) $P_{n}=C_{n} \cap \mathbb{Q}$ is dense in $C_{n}$,

(iv) if $t_{i} \in C_{i} \backslash P_{i}$ for $i \leq k$, then the sequence $\left(t_{1}, \ldots, t_{k}\right)$ is linearly independent over $\mathbb{Q}$.

Proof. First observe that it is enough to construct a sequence $\left(C_{n}\right)_{n=1}^{\infty}$ of subsets of $[0, \infty)$ satisfying conditions (ii)-(iv). Indeed, if $\left(C_{n}\right)$ is such a sequence, then we can obtain a sequence satisfying all conditions (i)-(iv) by replacing every $C_{n}$ by the set $\left\{q_{n} t: t \in C_{n}\right\}$ where $q_{n}$ is a suitably small positive rational number.

For every $A \subset \mathbb{N}$ we put $x_{A}=\sum_{n \in A} 1 / n$ !. The map $A \mapsto x_{A}$ is a homeomorphic embedding of $2^{\mathbb{N}}$ into $[0, \infty)$ (we treat $2^{\mathbb{N}}$ as a copy of the Cantor set). The continuity of this map is clear. In order to verify the injectivity we need to use the following standard estimate for every $k \geq 1$ :

$$
\begin{aligned}
\sum_{n=k+1}^{\infty} \frac{1}{n !} & =\frac{1}{(k+1) !}\left(1+\frac{1}{k+2}+\frac{1}{(k+2)(k+3)}+\ldots\right) \\
& <\frac{2}{(k+1) !} \leq \frac{1}{k !} .
\end{aligned}
$$

Now, take two distinct sets $A, B \subset \mathbb{N}$. Let $n$ be the smallest element of $(A \backslash B) \cup(B \backslash A)$; we can assume that $n \in A \backslash B$. Define $C=\{k \in A$ : $k<n\}=\{k \in B: k<n\}$. From (1) we obtain

$$
x_{A} \geq \sum_{k \in C} \frac{1}{k !}+\frac{1}{n !}>\sum_{k \in C} \frac{1}{k !}+\sum_{k=n+1}^{\infty} \frac{1}{k !} \geq x_{B} .
$$

Let $\left(N_{n}\right)_{n=1}^{\infty}$ be a partition of $\mathbb{N}$ into infinite sets. Define $C_{n}=\left\{x_{A}: A \subset\right.$ $\left.N_{n}\right\}$. Each $C_{n}$, being homeomorphic to $2^{N_{n}}$, is a topological copy of the Cantor set. Condition (iii) is also easy to verify. Namely, for every finite set 
$A \subset N_{n}$, the number $x_{A}$ is rational, and all these numbers form a dense subset of $C_{n}$. It remains to show that the sequence $\left(C_{n}\right)$ satisfies (iv).

Take $t_{i} \in C_{i} \backslash P_{i}$ for $i \leq k$ and let $t_{i}=x_{A_{i}}$ for some $A_{i} \subset N_{i}$. Obviously, every set $A_{i}$ is infinite since $t_{i}$ is irrational. Suppose that $\left(t_{1}, \ldots, t_{k}\right)$ is not linearly independent over $\mathbb{Q}$, i.e., there exist rational $q_{i}$ (at least one nonzero), $i \leq k$, such that $\sum_{i \leq k} q_{i} t_{i}=0$. We can write this sum in the following way:

$$
\sum_{n \in \mathbb{N}} \frac{a_{n}}{n !}=0,
$$

where each $a_{n}$ is equal to one of the numbers $q_{i}, i \leq k$, or 0 . Let $S_{j}=$ $\sum_{n \leq j} a_{n} / n$ ! for $j \geq 1$. Observe that for infinitely many $j$, we have $S_{j} \neq 0$ since infinitely many $a_{n}$ are nonzero. Let $q_{i}=p_{i} / r_{i}$, with integer $p_{i}, r_{i}$, and $r_{i}>0$. Put $M=\max \left\{\left|q_{i}\right|: i \leq k\right\}$ and $R=r_{1} \ldots r_{k}$. We take $j>2 M R$ such that $S_{j} \neq 0$. One can easily compute that $S_{j}=l /(j ! R)$, for some integer $l$. Therefore $\left|S_{j}\right| \geq 1 /(j ! R)$. This together with (2) implies that

$$
\left|\sum_{n=j+1}^{\infty} \frac{a_{n}}{n !}\right| \geq \frac{1}{j ! R} .
$$

But, on the other hand, using inequality (1) we can compute that

$$
\left|\sum_{n=j+1}^{\infty} \frac{a_{n}}{n !}\right| \leq \sum_{n=j+1}^{\infty} \frac{M}{n !}<\left(\frac{2 M}{j+1}\right) \frac{1}{j !}<\frac{1}{j ! R},
$$

which gives a contradiction.

3.3. Proposition. Let $\Phi=\left(\left(\varphi_{n}\right),\left(x_{n}\right)\right)$ be a biorthogonal system in a Fréchet space $X$ and let $F$ be a linear subspace of $X$ such that $Q_{\Phi} \subset F \subset$ $E_{\Phi}$. Then the space $F$ is not a $G_{\delta \sigma \delta}$-set in $X$.

Proof. Let $\left(s_{n}\right)$ be the sequence from Lemma 2.3 and let $\left(C_{n}\right)$ be the corresponding sequence of Cantor sets from Lemma 3.2. Denote by $S$ the subset of the product $\prod_{n \in \mathbb{N}} C_{n}$ consisting of all points $\left(x_{n}\right)$ such that $x_{n} \in P_{n}$ for all but finitely many $n$ (recall that $P_{n}=C_{n} \cap \mathbb{Q}$ ). From [CDM, Proposition $8.3(\mathrm{a})]$ it follows that $S$ is not a $G_{\delta \sigma \delta}$-set. Let $e: \prod_{n \in \mathbb{N}}\left[-s_{n}, s_{n}\right] \rightarrow X$ be a homeomorphic embedding from Lemma 2.3, i.e., $e\left(\left(t_{n}\right)\right)=\sum_{n \in \mathbb{N}} t_{n} x_{n}$. Then $e\left(\prod_{n \in \mathbb{N}} C_{n}\right)$ is a copy of the compact space $\prod_{n \in \mathbb{N}} C_{n}$ in $X$. Using the fact that $F$ is a linear subspace containing $Q_{\Phi}$ one can verify that $e(S) \subset F$. The inclusion $F \subset E_{\Phi}$ and property (iv) of the Cantor sets $C_{n}$ from Lemma 3.2 imply that $e\left(\prod_{n \in \mathbb{N}} C_{n}\right) \cap F \subset e(S)$. Therefore $e(S)=e\left(\prod_{n \in \mathbb{N}} C_{n}\right) \cap F$ is a closed copy of the space $S$ in $F$. This shows that $F$ is not a $G_{\delta \sigma \delta}$-set.

3.4. Corollary. The spaces $E_{\Phi}$ are $F_{\sigma \delta \sigma}$-sets which are not $G_{\delta \sigma \delta}$-sets. The same is true for the Banakh example $L$. 
Let us note that the upper estimate of the Borel class of $L$ (i.e., the fact $\left.L \in F_{\sigma \delta \sigma}\right)$ was established by Banakh [Ba, Lemma 3].

3.5. Remark. Banakh [Ba, Lemma 1] observed that $L$ and $L^{2}$ are homeomorphic. The same is true for the space $E_{\Phi}$, where $\Phi$ is the standard biorthogonal system in $\ell_{2}$. By [CDM, Proposition 8.3(a)], the countable products $E_{\Phi}^{\omega}$ and $L^{\omega}$ are $F_{\sigma \delta \sigma \delta}$-sets that are not $G_{\delta \sigma \delta \sigma}$-sets. It follows that $E_{\Phi}$ is not homeomorphic to $E_{\Phi}^{\omega}$, and $L$ is not homeomorphic to $L^{\omega}$. This observation can also be derived from the fact that $L$ is countable-dimensional [Ba, Lemma 2]. Repeating Banakh's argument one can show that, for the standard biorthogonal system in $\ell_{2}$, the (bigger) space $E_{\Phi}$ is also countabledimensional (similar reasoning also works for some other biorthogonal systems in separable Banach spaces).

\section{Failure of the $Z_{\sigma}$ property and of the Factor Theorem for} the spaces $E_{\Phi}$. We will use the following obvious fact:

4.1. LeMmA. Let $s_{1}, \ldots, s_{n}$ be a sequence of real numbers linearly independent over $\mathbb{Q}$. Then for every real $t \neq 0$ the sequence $\left(t s_{1}, \ldots, t s_{n}\right)$ is also linearly independent over $\mathbb{Q}$.

Proof. Fix $t \neq 0$ and suppose that $q_{1}\left(t s_{1}\right)+\ldots+q_{n}\left(t s_{n}\right)=0$ for some rationals $q_{1}, \ldots, q_{n}$. Dividing both sides of this equation by $t$ we obtain $q_{1} s_{1}+\ldots+q_{n} s_{n}=0$, hence by our assumption, $q_{1}=\ldots=q_{n}=0$.

4.2. Proposition. Let $\Phi=\left(\left(\varphi_{n}\right),\left(x_{n}\right)\right)$ be a biorthogonal system in a Fréchet space $X$ and let $F$ be a linear subspace of $X$ such that $Q_{\Phi} \subset F \subset E_{\Phi}$. Then the space $F$ contains a closed copy of $\sigma$.

Proof. Let $\left(s_{n}\right)$ be a sequence from Lemma 2.3. Take a sequence $\left(S_{n}\right)$ which is linearly independent over $\mathbb{Q}$ and such that $0<S_{n} \leq s_{n}$ for every $n$. For every $\left(t_{n}\right) \in[0,1]^{\mathbb{N}}$ define

$$
e\left(\left(t_{n}\right)\right)=\sum_{n=1}^{\infty}\left(\sum_{k=2^{n-1}}^{2^{n}-1}\left(t_{n} S_{k}\right) x_{k}\right) .
$$

By Lemma 2.3, the map $e$ is a homeomorphic embedding of $[0,1]^{\mathbb{N}}$ into $X$. Observe that since $Q_{\Phi} \subset F$, all vectors $x_{n}$ belong to $F$. Therefore we have $e(\sigma) \subset F$. For $m \in \mathbb{N}$, Lemma 2.3 implies that $\varphi_{k}\left(e\left(\left(t_{n}\right)\right)\right)=t_{m} S_{k}$ for $k=2^{m-1}, \ldots, 2^{m}-1$. Therefore, from Lemma 4.1, it follows that if $t_{m} \neq 0$ then $\operatorname{dim}_{\mathbb{Q}}\left(\left\{\varphi_{k}\left(e\left(\left(t_{n}\right)\right)\right): k \in \mathbb{N}\right\}\right) \geq 2^{m-1}$. Hence the inclusion $F \subset E_{\Phi}$ implies that $e\left([0,1]^{\mathbb{N}}\right) \cap F=e(\sigma)$. This shows that $e(\sigma)$ is a closed copy of $\sigma$ in $F$.

Modifying the above argument we obtain the following. 
4.3. Proposition. Let $\Phi=\left(\left(\varphi_{n}\right),\left(x_{n}\right)\right)$ be a biorthogonal system in a Fréchet space $X$ and let $F$ be a linear subspace of $X$ such that $Q_{\Phi} \subset$ $F \subset E_{\Phi}$. Then the space $F$ contains a closed linear subspace $V$ which is a $Z_{\sigma}$-space. Moreover, if the sequence $\left(\varphi_{n}\right)$ separates points of $X$, we can assume that $V$ is spanned by a countable subset.

Proof. Take a sequence $\left(S_{n}\right)$ of reals which is linearly independent over $\mathbb{Q}$. Put

$$
\begin{aligned}
& V=\left\{x \in F: \varphi_{k}(x)=S_{k} \varphi_{2^{n}}(x)\right. \\
& \left.\qquad \text { for all } n \in \mathbb{N} \text { and } k=2^{n}+1,2^{n}+2, \ldots, 2^{n+1}-1\right\}, \\
& W=\left\{x \in F: \varphi_{n}(x)=0 \text { for all } n \in \mathbb{N}\right\} .
\end{aligned}
$$

It is clear that both $V$ and $W$ are closed linear subspaces of $F$ and $W \subset V$. If the sequence $\left(\varphi_{n}\right)$ separates points of $X$, then obviously $W=\{0\}$. As above, by Lemma 4.1 , the sequence $\left(\varphi_{n}(x)\right)$ is eventually 0 for every $x \in V$. Therefore we have $V=\operatorname{span}\left(W \cup\left\{y_{n}: n \in \omega\right\}\right)$, where $y_{0}=x_{1}$ and

$$
y_{n}=x_{2^{n}}+\sum_{k=2^{n}+1}^{2^{n+1}-1} S_{k} x_{k} \quad \text { for } n \geq 1 .
$$

Take $V_{n}=\operatorname{span}\left(W \cup\left\{y_{i}: i=0,1, \ldots, n\right\}\right)$, the closed linear subspace of $V$. It is a standard fact that $V_{n}$, being of infinite codimension in $V$, is a $Z$-set in $V$. Clearly $V=\bigcup_{n \in \omega} V_{n}$.

4.4. Corollary. The Banakh example $L$ contains a closed infinitedimensional linear subspace $V$ spanned by a countable subset. In particular, $V$ is a topological copy of $\sigma$.

The proof of the next proposition follows closely the idea of Banakh (see [BRZ, pp. 166 and 210]).

4.5. Proposition. Let $\Phi=\left(\left(\varphi_{n}\right),\left(x_{n}\right)\right)$ be a biorthogonal system in a Fréchet space $X$. Then the subset $Q_{\Phi}$ is not contained in any $Z_{\sigma}$-set in $X$.

Fix a translation-invariant metric $d$ on $X$. We denote the closed ball in $(X, d)$ with center $x$ and radius $r$ by $\bar{B}(x, r)$. For every $t_{1}, \ldots, t_{n} \in \mathbb{R}$, we denote by $H\left(t_{1}, \ldots, t_{n}\right)$ the hyperplane $\left\{x \in X: \varphi_{i}(x)=t_{i}\right.$ for $i=$ $1, \ldots, n\}$.

We need the following two lemmas:

4.6. Lemma. The set $Q_{\Phi}$ is dense in $X$. Moreover, for every $q_{1}, \ldots, q_{n}$ $\in \mathbb{Q}$, the set $H\left(q_{1}, \ldots, q_{n}\right) \cap Q_{\Phi}$ is dense in $H\left(q_{1}, \ldots, q_{n}\right)$.

Proof. Fix $q_{1}, \ldots, q_{n} \in \mathbb{Q}, x \in H\left(q_{1}, \ldots, q_{n}\right)$ and $\varepsilon>0$. Using Lemma 2.3 we can find a sequence $\left(t_{k}\right)_{k=n+1}^{\infty}$ of reals such that the series $\sum_{k=n+1}^{\infty} t_{k} x_{k}$ is convergent to $y \in X$ and $d(0, y)<\varepsilon$. We may also require that $\varphi_{k}(x)+t_{k} \in \mathbb{Q}$ 
for every $k \geq n+1$. Then we have $d(x, x+y)<\varepsilon$ and, by Lemma 2.3, $\varphi_{k}(x+y) \in \mathbb{Q}$ for all $k$, hence $x+y \in Q_{\Phi}$. The same argument shows that $Q_{\Phi}$ is dense in $X$.

4.7. Lemma. For every $q_{1}, \ldots, q_{n} \in \mathbb{Q}, z \in H\left(q_{1}, \ldots, q_{n}\right)$ and $r>0$ the intersection $H\left(q_{1}, \ldots, q_{n}\right) \cap \bar{B}(z, r)$ is not a $Z$-set in $X$.

Proof. Let $Y=z+\operatorname{span}\left\{x_{1}, \ldots, x_{n}\right\}$ and let $p: X \rightarrow Y$ be the continuous projection given by $p(x)=z+\sum_{i=1}^{n} \varphi_{i}(x-z) x_{i}$. Observe that $H\left(q_{1}, \ldots, q_{n}\right)=p^{-1}(z)$. It is a well known consequence of Brouwer's Fixed Point Theorem that the identity map of a closed ball $B$ in $\mathbb{R}^{n}$ cannot be approximated by maps of $B$ into $\mathbb{R}^{n}$ that miss the center $c$ of $B$. Let $i: B \rightarrow Y \cap \bar{B}(z, r / 2)$ be an embedding such that $i(c)=z$. Then the above fact implies that $i$ cannot be approximated by maps $f: B \rightarrow X$ missing $H\left(q_{1}, \ldots, q_{n}\right) \cap \bar{B}(z, r)$, hence $H\left(q_{1}, \ldots, q_{n}\right) \cap \bar{B}(z, r)$ is not a $Z$-set in $X$. Indeed, if $f$ were such an approximation closer than $r / 2$ to $i$, then $f(B) \subset \bar{B}(z, r)$, so $f(B) \cap H\left(q_{1}, \ldots, q_{n}\right)=\emptyset$. Therefore $p \circ f(B) \subset Y \backslash\{z\}$. It is clear that if $f$ were sufficiently close to $i$ then also $p \circ f$ would be close to $p \circ i=i$. This contradicts the above property of $B$.

Proof of Proposition 4.5. Aiming at a contradiction, assume that $\left(A_{n}\right)_{n \in \mathbb{N}}$ is a sequence of $Z$-sets in $X$ such that $Q_{\Phi} \subset \bigcup_{n \in \mathbb{N}} A_{n}$. By induction we will construct, for every $n \in \mathbb{N}$, a rational $q_{n}$, a point $z_{n} \in X$, and a real number $r_{n} \in(0,1 / n)$ such that:

(a) $z_{n} \in H\left(q_{1}, \ldots, q_{n}\right) \cap Q_{\Phi}$,

(b) $\bar{B}\left(z_{n}, r_{n}\right) \cap A_{n}=\emptyset$,

(c) $\bar{B}\left(z_{n}, r_{n}\right) \subset \bar{B}\left(z_{n-1}, r_{n-1}\right)$ (for $\left.n>1\right)$.

We start the construction by taking $q_{1}=0$. Lemma 4.7 implies that $H\left(q_{1}\right)$ is not a $Z$-set in $X$, therefore the set $H\left(q_{1}\right) \backslash A_{1}$ is nonempty. Since $A_{1}$ is closed, Lemma 4.6 implies the existence of $z_{1} \in H\left(q_{1}\right) \cap Q_{\Phi}$ and $r_{1} \in(0,1)$ such that $\bar{B}\left(z_{1}, r_{1}\right) \cap A_{1}=\emptyset$.

Now, suppose that $n>1$ and we have chosen $q_{i}, z_{i}$ and $r_{i}$ satisfying conditions (a)-(c), for $i<n$. We put $q_{n}=\varphi_{n}\left(z_{n-1}\right)$, so $z_{n-1} \in H\left(q_{1}, \ldots, q_{n}\right)$. By Lemma 4.7 , the intersection $H\left(q_{1}, \ldots, q_{n}\right) \cap \bar{B}\left(z_{n-1}, r_{n-1}\right)$ is not a $Z$-set in $X$, hence we have $\left(H\left(q_{1}, \ldots, q_{n}\right) \cap \bar{B}\left(z_{n-1}, r_{n-1}\right)\right) \backslash A_{n} \neq \emptyset$. Then, applying Lemma 4.6, we can find $z_{n} \in H\left(q_{1}, \ldots, q_{n}\right) \cap Q_{\Phi}$ and $r_{n} \in(0,1 / n)$ such that $\bar{B}\left(z_{n}, r_{n}\right) \subset \bar{B}\left(z_{n-1}, r_{n-1}\right) \backslash A_{n}$.

The inequality $r_{n}<1 / n$ and condition (c) imply that the intersection $\bigcap_{n \in \mathbb{N}} \bar{B}\left(z_{n}, r_{n}\right)$ contains a unique element $z$. Obviously $z$ is the limit of the sequence $\left(z_{n}\right)$. By condition (a) we have $\varphi_{n}(z)=q_{n}$ for every $n$; therefore $z \in Q_{\Phi}$. On the other hand, condition (b) implies that $z \in \bar{B}\left(z_{n}, r_{n}\right) \subset$ $X \backslash A_{n}$ for every $n$, hence $z \notin \bigcup_{n \in \mathbb{N}} A_{n}$, which is the required contradiction. 
4.8. Corollary. Let $\Phi=\left(\left(\varphi_{n}\right),\left(x_{n}\right)\right)$ be a biorthogonal system in a Fréchet space $X$. Then the set $\left(Q_{\Phi}\right)^{\omega}$ is not contained in any $Z_{\sigma}$-subset of $X^{\omega}$.

Proof. It is enough to observe that $\left(Q_{\Phi}\right)^{\omega}=Q_{\Psi}$ for a suitable biorthogonal system $\Psi$ in a Fréchet space $X^{\omega}$. Namely, let $\left\{\left(m_{k}, n_{k}\right): k \in \mathbb{N}\right\}$ be an enumeration of $\mathbb{N} \times \omega$. Put $\psi_{k}\left(\left(z_{i}\right)_{i}\right)=\varphi_{m_{k}}\left(z_{n_{k}}\right)$ for $\left(z_{i}\right)_{i} \in X^{\omega}$ and $k \in \mathbb{N}$. Let $y^{k}=\left(y_{i}^{k}\right)_{i} \in X^{\omega}$, where $y_{i}^{k}=x_{m_{k}}$ for $i=n_{k}$, and $y_{i}^{k}=0$ for $i \neq n_{k}$. Then the system $\Psi=\left(\left(\psi_{k}\right)_{k},\left(y^{k}\right)_{k}\right)$ has the required property.

4.9. Corollary. For every biorthogonal system $\Phi$ in a Fréchet space $X$, the spaces $E_{\Phi}$ and $\left(E_{\Phi}\right)^{\omega}$ are not $Z_{\sigma}$-spaces. Also the space $L^{\omega}$ is not a $Z_{\sigma}$-space.

Now, we are in a position to formulate our main result.

4.10. Theorem. For every Fréchet space $X$ with a biorthogonal system $\Phi$, the linear subspace $E_{\Phi}$ of $X$ is of exact Borel class $F_{\sigma \delta \sigma}$ and contains a retract $R$, homeomorphic to $\sigma$, such that $R \times E_{\Phi}^{\omega}$ is not homeomorphic to $E_{\Phi}^{\omega}$. In particular, the spaces $R \times E_{\Phi}$ and $E_{\Phi}$ are not homeomorphic (cf. Remark 3.5).

4.11. Remark. The Banakh example $L$ is another space that is of exact Borel class $F_{\sigma \delta \sigma}$ and contains a retract $R$ such that $R \times L^{\omega}$ is not homeomorphic to $L^{\omega}$.

5. $Z_{\sigma}$-spaces which are powers of non- $Z_{\sigma}$-spaces. It is an obvious fact that the product of a $Z_{\sigma}$-space and any space is a $Z_{\sigma}$-space. Examples show that products of non- $Z_{\sigma}$-spaces might be $Z_{\sigma}$-spaces. Below, we provide examples of such spaces that carry some additional structure. However, we do not know whether, given a normed linear space $E$ which is not a $Z_{\sigma^{-}}$ space, its power $E^{n}$ (finite or countable) is not a $Z_{\sigma}$-space. Therefore, in the previous section, knowing that $E_{\Phi}$ (or $L$ ) was not a $Z_{\sigma}$-space, we had to verify the same property for $E_{\Phi}^{\omega}$ (resp., $\left.L^{\omega}\right)$.

5.1. Example. There exists a $\sigma$-compact convex subset $X$ of $\ell_{2}$ such that

(1) $X$ is not a $Z_{\sigma}$-space, and

(2) $X \times X$ is a $Z_{\sigma}$-space.

The space $X$ will be the one described in $[\mathrm{CuDM}]$. Let $W$ be a wild (i.e., not a $Z$-set) Cantor set in the infinite-dimensional compact convex ellipsoid

$$
M=\left\{\left(x_{i}\right) \in \ell_{2}: \sum_{i=1}^{\infty} i^{2} x_{i}^{2} \leq 1\right\},
$$


a topological Hilbert cube. Let $M_{\text {core }}=\left\{\left(x_{i}\right) \in \ell_{2}: \sum_{i=1}^{\infty} i^{2} x_{i}^{2}<1\right\}$, a topological copy of $\Sigma=\left\{\left(x_{i}\right) \in[-1,1]^{\omega}: \sup \left|x_{i}\right|<1\right\}$. Then $X=M_{\text {core }} \cup$ $W$ is a $\sigma$-compact convex subset of $\ell_{2}$, and $W$ is not a $Z$-set in $X$. Hence, $X$ is not a $Z_{\sigma}$-space (see [CuDM, Lemma 2.4]).

Now, we will show that $X \times X$ is a $Z_{\sigma}$-space. Since every compactum $L \subset X$ with $L \cap W=\emptyset$ is a $Z$-set, it is enough to show that $A=W \times W$ is a $Z$-set in $X \times X$. By a result of Kroonenberg [Kr], it is enough to show that there is a base $\mathcal{U}$ of homotopically trivial open sets in $X \times X$ such that, for every $U \in \mathcal{U}, U \backslash A$ is homologically trivial, path-connected and simply connected. Since $A$ is finite-dimensional (actually, 0-dimensional), the homological triviality and the path-connectedness of $U \backslash A$ easily follow for any homotopically trivial open subset $U$ of $X \times X$ (see [D2]). Let $U$ be of the form $V \times V$, where $V$ is a homotopically trivial open subset of $X$. It suffices to show that $V \times V \backslash A$ is simply connected. This follows from Corollary 1 in $[\mathrm{BT}]$. For the sake of completeness we decided to include a short proof that mimics a reasoning from $[\mathrm{Li}]$.

Let $f: S^{1} \rightarrow V \times V \backslash A$ be a map of the circle $S^{1}$. Setting $f=(g, h)$, where $g, h: S^{1} \rightarrow V$ are the components of $f$, we see that $g^{-1}(W)$ and $h^{-1}(W)$ are disjoint compacta in $S^{1}$. Let $\left\{C_{1}, \ldots, C_{m}\right\}$ be a family of pairwise disjoint arcs of $S^{1}$ such that $g^{-1}(W) \subset \bigcup_{j=1}^{m}$ int $C_{j}$ and $h^{-1}(W) \cap \bigcup_{j=1}^{m} C_{j}=\emptyset$. Since $V \backslash W$ is path-connected and $V$ is homotopically trivial, there exists a homotopy $\varphi_{1}$ joining $g$ and $\varphi_{1}^{1}$ in $V$ relative to $S^{1} \backslash \bigcup_{j=1}^{m} \operatorname{int} C_{j}$ such that $\operatorname{im}\left(\varphi_{1}^{1}\right) \cap W=\emptyset$. Let $\varphi_{2}$ be a homotopy in $V$ joining $h$ to a constant map into $V \backslash W$, and let $\varphi_{3}$ be a homotopy in $V$ joining $\varphi_{1}^{1}$ to a constant map into $V \backslash W$. Then, combining the homotopies $\Phi_{1}=\left(\varphi_{1}, h\right), \Phi_{2}=\left(\varphi_{1}^{1}, \varphi_{2}\right)$, and $\Phi_{3}=\left(\varphi_{3}, \varphi_{2}^{1}\right)$, we can join $f$ to a constant map into $V \times V \backslash W \times W$.

It is impossible to find a convex space $X$ which is a countable union of finite-dimensional compacta and satisfies the above conditions (see [D2]). However, there exists an absolute retract $X$ that is nowhere locally compact such that $X$ is not a $Z_{\sigma}$-space but $X \times X$ is a $Z_{\sigma}$-space, and $X$ is a countable union of finite-dimensional compacta. Again the space $X$ was described in $[\mathrm{CuDM}]$. Namely, $X=M_{f} \cup W$, where $M_{f}=\left\{\left(x_{i}\right) \in M: x_{i}=0\right.$ a.e. $\}$, and $M$ and $W$ are as above. Clearly, $X$ is a countable union of finite-dimensional compacta. Moreover, the above argument can be repeated to obtain the claimed property of $X$.

Below, we present an easier example of an absolute retract $X$ which is not a $Z_{\sigma}$-space, but $X^{\omega}$ is a $Z_{\sigma}$-space.

5.2. Example. There exists a $\sigma$-compact absolute retract $X$ such that

(1) $X^{n}$ is not a $Z_{\sigma}$-space for any $n \in \mathbb{N}$, and

(2) $X^{\omega}$ is a $Z_{\sigma}$-space. 
Define $X=(\sigma \times\{0\}) \cup(\{\overline{0}\} \times[0,1])$ as a subset of $Q \times[0,1]$, where $Q=[0,1]^{\omega}, \sigma=\left\{\left(x_{i}\right) \in Q: x_{i}=0\right.$ a.e. $\}$, and $\overline{0}=(0,0, \ldots) \in Q$. Represent the space $\sigma \times\{0\} \backslash\{(\overline{0}, 0)\}$ as a countable union of $Z$-sets $A_{n}$ in $\sigma \times\{0\}$, where each $A_{n}$ is compact and contained in $\left\{\left(x_{i}\right) \in \sigma: x_{i}=0\right.$ for $\left.i>n\right\} \times\{0\}$. Then $X^{\omega}$ can be represented as the union of

$$
B_{0}=(\{\overline{0}\} \times[0,1]) \times(\{\overline{0}\} \times[0,1]) \times \ldots \subset X \times X \times \ldots
$$

and

$$
B_{n}^{k}=X^{k} \times A_{n} \times X \times X \times \ldots \subset X^{k} \times X \times X \times \ldots=X^{\omega}
$$

for $k, n \in \omega$. Since each $A_{n}$ is a $Z$-set in $X$, it is easy to see that each $B_{n}^{k}$, $k, n=0,1, \ldots$, is a $Z$-set in $X$. Moreover, clearly, $B_{0}$ is a $Z$-set in $X$. Hence, $X^{\omega}$ is a $Z_{\sigma}$-space.

For any $n \in \mathbb{N}$, the compact set $(\{\overline{0}\} \times[0,1])^{n} \subset X^{n}$ has a nonempty interior in $X^{n}$, hence $X^{n}$ is not of the first category in itself. Consequently, it is not a $Z_{\sigma}$-space.

In general, if an absolute retract $X$ contains an open subset that is a $Z_{\sigma}$-space in itself, then $X^{\omega}$ is a $Z_{\sigma}$-space. However, this provides nontrivial information only if $X$ is nonhomogeneous, because if $X$ is homogeneous and satisfies the above condition, then $X$ is a $Z_{\sigma}$-space itself (and therefore $X^{\omega}$ is a $Z_{\sigma}$-space as well). It would be interesting to find conditions ensuring that an infinite-dimensional absolute retract $X$ is not a $Z_{\sigma}$-space, and this property carries over to $X^{2}, X^{n}$ or $X^{\omega}$. Here, the homogeneity of $X$ seems to be essential; the first case to consider is when $X$ is a pre-Hilbert space.

6. Remarks and comments on the Factor Theorem. Let us start with the construction of R. Pol mentioned in the Introduction, which we include with his kind permission.

6.1. Example (R. Pol). There exists a linear subspace $E$ of the Hilbert space $\ell_{2}$ which is a Baire space and contains a closed linear subspace $R$ homeomorphic to the space $\sigma$. The subspace $R$ is a retract of $E$ such that $R \times E^{\omega}$ is not homeomorphic to $E^{\omega}$ (hence $R \times E$ is not homeomorphic to $E)$.

For technical reasons we will construct our space $E$ in $\ell_{2} \times \ell_{2}$, an isometric copy of $\ell_{2}$. Let $\pi_{1}: \ell_{2} \times \ell_{2} \rightarrow \ell_{2}$ denote the projection onto the first axis. Let $\mathcal{C}$ be the family of all copies $C$ of the Cantor set in $\ell_{2} \times \ell_{2}$ such that $\pi_{1}$ is injective on $C$ and $\pi_{1}(C)$ is linearly independent (over $\mathbb{R}$ ). The family $\mathcal{C}$ has the cardinality of the continuum, hence we can enumerate it as $\left\{C_{\alpha}\right.$ : $\left.\alpha<2^{\omega}\right\}$.

Using transfinite induction we can choose vectors $x_{\alpha} \in C_{\alpha}$, for $\alpha<2^{\omega}$, such that $x_{\alpha} \notin \operatorname{span}\left(\left\{x_{\beta}: \beta<\alpha\right\} \cup\{0\} \times \ell_{2}\right)$. Then we put $E=\operatorname{span}\left(\left\{x_{\alpha}\right.\right.$ : $\left.\left.\alpha<2^{\omega}\right\} \cup\left\{\left(0, e_{n}\right): n \in \mathbb{N}\right\}\right)$. The choice of the vectors $x_{\alpha}$ guarantees that 
$R=E \cap\left(\{0\} \times \ell_{2}\right)=\operatorname{span}\left\{\left(0, e_{n}\right): n \in \mathbb{N}\right\}$, and consequently, $R$ is a closed linear subspace of $E$ homeomorphic to $\sigma$. It remains to prove that $E$ is a Baire space because then $E^{\omega}$ is also a Baire space by a theorem of Oxtoby [Ox]. Since $E$ is homogeneous it is enough to show that $E$ is of the second category in $\ell_{2} \times \ell_{2}$. If $E$ were of the first category in $\ell_{2} \times \ell_{2}$, then Proposition 2.1 from [vMP] would imply the existence of a copy of a Cantor set $C \subset\left(\ell_{2} \times \ell_{2}\right) \backslash E$ such that $C \in \mathcal{C}$. This would contradict the fact that $E$ intersects every set $C$ in $\mathcal{C}$.

Recent achievements in the theory of absorbing sets allow us to formulate the following version of the Factor Theorem.

6.2. Proposition. Let $E$ be a separable metric linear $Z_{\sigma}$-space. Assume that $E$ is an $A R$ and $E$ is homeomorphic to its own square $E^{2}$. Then, for every retract $R$ of $E$, the product $R \times E$ is homeomorphic to $E$.

Proof. Apply [BRZ, 4.2.1 and 5.3.17] and [BM, 5.4].

We say that a metrizable space $X$ is $\sigma$-complete if $X=\bigcup_{n \in \omega} X_{n}$, where each $X_{n}$ is completely metrizable and closed in $X$. Clearly, every $\sigma$-compact metrizable space is $\sigma$-complete. Hence the following fact yields an affirmative answer to Problem 1.1 for the $\sigma$-compact case (as indicated in the Introduction).

6.3. TheOrem. Let $G$ be a separable $\sigma$-complete topological group that is an absolute retract. Then, for every retract $R$ of $G$, the spaces $R \times G^{\omega}$ and $G^{\omega}$ are homeomorphic.

Proof. Obviously, we have $G=\{e\}$ if $G$ is compact (by the fixed point property of compact absolute retracts). Therefore we may assume that $G$ is noncompact.

If $G$ is completely metrizable then both $R \times G^{\omega}=(R \times G) \times G^{\omega}$ and $G^{\omega}$, being countable products of separable completely metrizable noncompact AR's, are homeomorphic to $\mathbb{R}^{\omega}$ (cf. [Tor3]).

In the case when $G$ is not completely metrizable, by Theorem 4.2 .8 from [BRZ], $G$ is a $Z_{\sigma}$-space. Since $G$ is an absolute $F_{\sigma \delta}$-set, Corollary 2.7 from [DM2] implies that $G^{\omega}$ is homeomorphic to $\sigma^{\omega}$. Now, by a result of [BM], for a retract $R$ of $\sigma^{\omega}$, the product $R \times \sigma^{\omega}$ is homeomorphic to $\sigma^{\omega}$.

It is worthwhile to examine Problem 1.1 in case the assumption that $E$ is a linear space is dropped, and instead it is imposed that $E$ is an absolute retract. In such a case, if $E$ is compact, then either $E$ is a singleton, or, by known results, $E^{\omega}$ is homeomorphic to the Hilbert cube. In both instances, for a retract $R$ of $E$, we have $R \times E^{\omega}$ homeomorphic to $E^{\omega}$. If $E$ is completely metrizable and noncompact, then $E^{\omega}$ is known to be homeomorphic to an infinite-dimensional Hilbert space [Tor3], and again the answer to 
our problem is affirmative. For the case where $E$ is an incomplete absolute retract, we have the following fact that complements Theorem 6.3 ; similarly to Proposition 6.2 , it can be easily derived from results on absorbing sets.

6.4. Proposition. Let $E$ be a separable absolute retract space that is a $Z_{\sigma}$-space. Then, for every retract $R$ of $E, R \times E^{\omega}$ is homeomorphic to $E^{\omega}$. Actually, it is enough to require that $E^{\omega}$ is a $Z_{\sigma}$-space.

Proof. This is a consequence of [BM, 2.5] (use also the results of [DM2], in particular, Lemma 2.2 therein). Namely, those results show that $E^{\omega}$ is an absorbing set for the class of all its closed subsets. Hence, the assertion follows from [BM, 5.4].

6.5. QueStion. Is a separable noncomplete normed linear space $E \in F_{\sigma \delta}$ necessarily a $Z_{\sigma}$-space?

If the answer is affirmative then, by 6.4 , Problem 1.1 also has an affirmative solution for the $F_{\sigma \delta}$-case.

The $Z_{\sigma}$ property is a stronger version of the first category property. When we try to relax the $Z_{\sigma}$ property, the Baire spaces with a nice local structure can serve as examples answering 1.1 in the negative. The following example shows that the $Z_{\sigma}$ property cannot be relaxed even if we assume the convexity of a set and, additionally, even if the set is homogeneous.

6.6. Example. Consider $Y=\sigma \times\{0\} \cup Q \times(0,1]$, a subspace of $Q \times[0,1]$. The space $Y$ is a Baire space because it contains the Baire space $Q \times(0,1]$ as a dense subset. It follows that $Y^{\omega}$ is also a Baire space. Letting $R=\sigma \times\{0\}$ we see that $R$ is a closed subset of $Y$ and, consequently, is a retract of $Y$. Since $R \times Y^{\omega}$ is of the first category, it cannot be homeomorphic to $Y^{\omega}$. Both $Y$ and $R$ are convex sets. Furthermore, the space $E=Y^{\omega}$ is a so-called $F_{\sigma \delta}$-coabsorbing set (see the definition in [BRZ, p. 43]); hence, not only is $E$ topologically homogeneous, but also any homeomorphism between any two $Z$-sets (in particular, any two compacta) in $E$ extends to an autohomeomorphism of $E$ (see [BRZ, p. 49]). Clearly, the convex set $E$ contains a closed convex copy of $R$. Obviously, the product $E^{\omega}$ can be identified with $E$.

\section{References}

[Ba] T. Banakh, Some properties of the linear hull of the Erdös set in $\ell^{2}$, Bull. Polish Acad. Sci. Math. 47 (1999), 385-392.

[BRZ] T. Banakh, T. Radul and M. Zarichnyi, Absorbing Sets in Infinite-Dimensional Manifolds, VNTL Publishers, Lviv, 1996. 
[BT] T. Banakh and Kh. Trushchak, $Z_{n}$-sets and the disjoint $n$-cells property in products of ANR's, Mat. Stud. 13 (2000), 74-78.

[BP] C. Bessaga and A. Pełczyński, Selected Topics in Infinite-Dimensional Topology, Polish Sci. Publ., Warszawa, 1975.

[BM] M. Bestvina and J. Mogilski, Characterizing certain incomplete infinite-dimensional retracts, Michigan Math. J. 33 (1986), 291-313.

[CDM] R. Cauty, T. Dobrowolski and W. Marciszewski, A contribution to the topological classification of the spaces $C_{\mathrm{p}}(X)$, Fund. Math. 142 (1993), 269-301.

$[\mathrm{CuDM}]$ D. Curtis, T. Dobrowolski and J. Mogilski, Some applications of the topological characterizations of the sigma-compact spaces $l_{2}^{f}$ and $\Sigma$, Trans. Amer. Math. Soc. 284 (1984), 837-846.

[D1] T. Dobrowolski, Examples of topological groups homeomorphic to $l_{2}^{f}$, Proc. Amer. Math. Soc. 98 (1986), 303-311.

[D2] - , The compact Z-set property in convex sets, Topology Appl. 23 (1986), 163172.

[DM1] T. Dobrowolski and J. Mogilski, Problems on topological classification of incomplete metric spaces, in: Open Problems in Topology, J. van Mill and G. M. Reed (eds.), North-Holland, Amsterdam, 1990, 409-429.

[DM2] - - - Certain sequence and function spaces homeomorphic to the countable product of $l_{f}^{2}$, J. London Math. Soc. 45 (1992), 556-576.

[Ge] R. Geoghegan, Open problems in infinite-dimensional topology, Topology Proc. 4 (1979), 287-330.

[Kr] N. Kroonenberg, Characterization of finite dimensional Z-sets, Proc. Amer. Math. Soc. 43 (1974), 421-427.

[Ku] M. Kunzinger, Barrelledness, Baire-like- and (LF)-spaces, Longman Sci. Tech., 1993.

[Li] V. T. Liem, A counterexample in $\ell_{2}$-manifold theory, Proc. Amer. Math. Soc. 73 (1979), 119-120.

[LT] J. Lindenstrauss and L. Tzafriri, Classical Banach Spaces I, Springer, Berlin, 1997.

[MvM] W. Marciszewski and J. van Mill, An example of $t_{p}^{*}$-equivalent spaces which are not $t_{p}$-equivalent, Topology Appl. 85 (1998), 281-285.

[vM] J. van Mill, Infinite-Dimensional Topology. Prerequisites and Introduction, North-Holland, Amsterdam, 1989.

[vMP] J. van Mill and R. Pol, The Baire category theorem in products of linear spaces and topological groups, Topology Appl. 22 (1986), 267-282.

[Ox] J. Oxtoby, Cartesian products of Baire spaces, Fund. Math. 49 (1961), 157-166.

[PCB] P. Pérez Carreras and J. Bonet, Barrelled Locally Convex Spaces, North-Holland Math. Stud. 131, North-Holland, 1987.

[Tor1] H. Toruńczyk, Absolute retracts as factors of normed linear spaces, Fund. Math. 86 (1974), 53-67.

[Tor2] -, On Cartesian factors and the topological classification of linear metric spaces, ibid. 88 (1975), 71-86.

[Tor3] —, Characterizing Hilbert space topology, ibid. 111 (1981), 247-262.

[Va] M. Valdivia, Topics in Locally Convex Spaces, North-Holland Math. Stud. 67, North-Holland, 1982. 
[We] J. E. West, Problems in infinite-dimensional topology, in: Open Problems in Topology, J. van Mill and G. M. Reed (eds.), North-Holland, Amsterdam, 1990, $523-597$.

Department of Mathematics

Pittsburg State University

Pittsburg, KS 66762, U.S.A.

E-mail: tdobrowo@mail.pittstate.edu

Current address:

Department of Mathematics

University of Missouri-Columbia

Columbia, MO 65211, U.S.A.
Institute of Mathematics University of Warsaw

Banacha 2

02-097 Warszawa, Poland

E-mail: wmarcisz@mimuw.edu.pl

Current address:

Division of Mathematics and Computer Science

Faculty of Sciences Vrije Universiteit

De Boelelaan $1081^{\mathrm{a}}$

1081 HV Amsterdam, The Netherlands

Received 16 July 2001;

in revised form 13 June 2002 